\title{
L'espace créateur. Urbanité et théâtralité dans le Londres de Shakespeare
}

Andreas Mahler

\section{(2) OpenEdition}

\section{Journals}

\section{Édition électronique}

URL : http://journals.openedition.org/shakespeare/1627

DOI : 10.4000/shakespeare.1627

ISSN : 2271-6424

Éditeur

Société Française Shakespeare

\section{Édition imprimée}

Date de publication : 1 mars 2011

Pagination : 185-205

ISBN : 2-9521475-7-4

\section{Référence électronique}

Andreas Mahler, «L'espace créateur. Urbanité et théâtralité dans le Londres de Shakespeare », Actes des congrès de la Société française Shakespeare [En ligne], 28 | 2011, mis en ligne le 15 février 2011, consulté le 01 mai 2019. URL : http://journals.openedition.org/shakespeare/1627 ; DOI : 10.4000/ shakespeare. 1627 


\section{Shakespeare et la Cité}

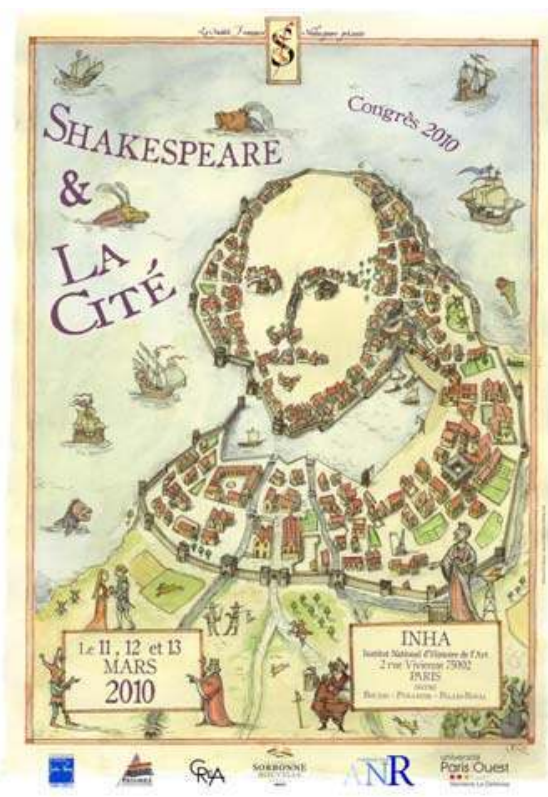

actes du Congrès

organisé par la

SOCIÉTÉ FRANÇAISE SHAKESPEARE

les 11,12 et 13 mars 10

textes réunis par

Pierre KAPITANIAK

sous la direction de

Dominique GoY-BLANQUET 
COUVERTURE :

Edouard Lekston 2010

conception graphique et logo

Pierre Kapitaniak

\section{(C) 2011 Société Française Shakespeare Institut du Monde Anglophone} Université de Paris III - Sorbonne Nouvelle 5 rue de l'École de Médecine 75006 Paris

www.societefrancaiseshakespeare.org réservés pour tous les pays 


\title{
L'ESPACE CRÉATEUR. URBANITÉ ET THÉÂTRALITÉ DANS LE LONDRES DE SHAKESPEARE
}

\author{
Andreas MAHLER
}

\begin{abstract}
Cette contribution prend son point de départ dans les réflexions récentes sur la notion d'espace pour explorer le Londres de 1600 comme un espace urbain caractérisé par une multiplicité de perspectives menant à une sorte de théâtralité sociale qui peut être considérée comme une des bases décisives pour l'explosion créatrice typique à l'époque de Shakespeare.
\end{abstract}

The contribution takes up recent reflections on the notion of space in order to explore late sixteenth-century London as an urban space characterized by a multiplicity of perspectives triggering off a kind of social theatricality that can be seen as one of the decisive impulses for the explosion of creativity which has been considered typical of the Age of Shakespeare.

\section{Lieux et espaces}

T es villes, comme l'écrivait Fernand Braudel, sont autant de transformateurs électriques : elles augmentent les tensions,

" elles précipitent les échanges, elles brassent sans fin la vie des hommes ${ }^{1}$. Ce que j'ai l'intention de proposer dans les réflexions suivantes portant sur l'aspect créateur du Londres prémoderne (ill. 1), c'est un rapprochement entre la « théorie de l'espace » d'une part, et la notion de «constellation » de l'autre, et je vais commencer par la distinction entre «lieu» et « espace $^{2} »$. C'est, entre autres, Michel de Certeau, qui, dans ses Arts de faire, a développé cette distinction à partir de celle entre la "stratégie », notion désignant l'intérêt sémantique de toute une société, et la «tactique», contre-courant désignant les options d'une pragmatique individuelle. Par conséquent, la stratégie se situe dans des lieux concrets, réalisables, tandis que les tactiques trouvent leur place dans des lieux qui ne leur appartiennent

\footnotetext{
${ }^{1}$ Fernand Braudel, Civilisation matérielle, économie et capitalisme, $X V^{e}-X V I I I^{e}$ siècle, 3 tomes, tome 1 : Les structures du quotidien : le possible et l'impossible, Paris, Armand Colin, 1979, p. 421.

2 Pour une conjoncture heureuse d'études shakespeariennes avec ce qu'on appelle la "théorie française », voir, par exemple, Richard Wilson, Shakespeare in French Theory. King of Shadows, Londres, Routledge, 2007 ; pour une version plus détaillée des réflexions suivantes, voir aussi Andreas Mahler, "Beginning in the middle. Strategie und Taktik an den Inns of Court », Zeitsprünge, no 13, 2009, p. 1-22.
} 


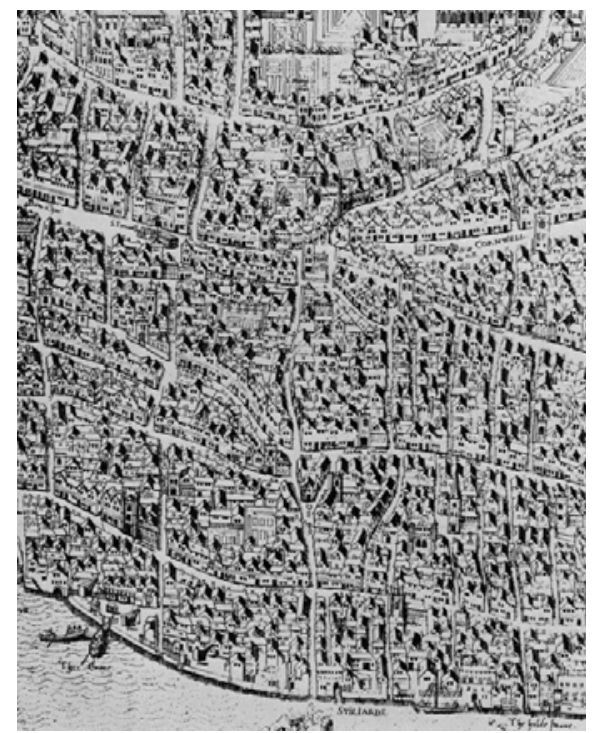

\section{Ill. 1: Rues de Londres (détail) - Felix Barker \& Peter Jackson, London. 2000 years of a city and its people, Londres, Cassell, 1974, p. 70-71.}

pas, des lieux usurpés, appropriés, c'est-à-dire dans un espace mis en jeu par un individu poursuivant ses propres intérêts :

J'appelle stratégie le calcul (ou la manipulation) des rapports de forces qui devient possible à partir du moment où un sujet de vouloir et de pouvoir (une entreprise, une armée, une cité, une institution scientifique) est isolable. Elle postule un lieu susceptible d'être circonscrit comme un propre et d'être la base d'où gérer les relations avec une extériorité de cibles ou de menaces (les clients ou les concurrents, les ennemis, la campagne autour de la ville, les objectifs et objets de la recherche, etc.). [...] [J]'appelle tactique l'action calculée que détermine l'absence d'un propre. Alors aucune délimitation de l'extériorité ne lui fournit la condition d'une autonomie. La tactique n'a pour lieu que celui de l'autre. Aussi doit-elle jouer avec le terrain qui lui est imposé tel que l'organise la loi d'une force étrangère ${ }^{3}$.

Certeau, on le sait bien, est fonctionnaliste : son concept de base est celui d'une sémantique sociale qui ne se réalise qu'à travers des actes d'une pragmatique individuelle ; ce qu'il vise est une structure qui

3 Michel de Certeau, L’invention du quotidien. 1. Arts de faire, éd. Luce Giard, coll. Folio Essais, Paris, Gallimard, 1990, p. 59sq. (c'est Certeau qui souligne), voir aussi p. XLVIsq. 
n'est visible qu'au moment de sa réalisation fonctionnelle. Par conséquent, une société semble se constituer à partir d'idées collectives qui s'articulent dans les moments concrets du comportement humain ; elle se définit par un imaginaire qui n'existe que dans sa réalisation ponctuelle concrète. Cela correspond à l'idée d'une simultanéité entre l'acte individuel constituant à la fois la société et le système social formant l'individu, le champ où tout cela a lieu étant le champ du symbolique. Voilà le lieu d'une « institution imaginaire de la société4. »

On voit l'analogie avec le modèle saussurien de "langue » et «parole ». L'analyse de Michel de Certeau est structurale (sinon structuraliste); mais elle l'est au sens mathématique ou logique du terme, tel qu'il se trouve propagé, par exemple, par le structuralisme génétique d'un Jean Piaget, c'est-à-dire non comme modèle de surfaces et de profondeurs, mais comme domaine de la relationalité5. Et on peut trouver cela chez Certeau lui-même: «la relation (toujours sociale) détermine ses termes, et non l'inverse », explique-t-il tout au début de son «Introduction générale » en poursuivant : "chaque individualité est le lieu où joue une pluralité incohérente (et souvent contradictoire) de ses déterminations relationnelles ${ }^{6}$. " La relation détermine donc les éléments, mais sans éléments, pas de description de la relation; l'imaginaire détermine l'utilisation du réel (du matériel), mais seul le réel, et son utilisation, sont ce qui est analysable.

Le modèle proposé est donc pour ainsi dire celui d'un structuralisme «sans fond». Ce que nous comprenons par une « société » n'est rien qu'une trace, une « trajectoire" » d'appropriations imaginaires: un réseau de trajectoires, leur combinaison, leur croisement, leur confusion, leur parallélisme; et son analyse se restreint nécessairement à une certaine monodimensionnalité : seuls nous sont accessibles les actes observables, leur groupement, leur succession. Voilà «l'invention du quotidien", sa «fiction ${ }^{8}$ ». La manière dont procède cette invention (permanente) du quotidien se caractérise à son tour par la relation entre l'imaginaire social et l'acte

4 Pour cette notion, voir Cornelius Castoriadis, L’institution imaginaire de la société, coll. Points, Paris, Seuil, 1999.

5 Voir Jean Piaget, Le structuralisme, coll. Que sais-je ?, Paris, PUF, 1968.

${ }^{6}$ Certeau, op. cit., p. xxxvsq.

7 Ibid., p. XLvsq., voir aussi p. 58 sq.

${ }^{8}$ Voir Wolfgang Iser, The Fictive and the Imaginary. Charting Literary Anthropology, trad. David Henry Wilson, Baltimore, MD, Johns Hopkins U. P., 1993. 
pragmatique individuel : si ces deux derniers se réaffirment dans une relation d'équivalence, on a ce que Certeau appelle la « stratégie »; s'ils se contredisent dans une relation d'opposition, ce qui règne c'est la «tactique ». Si donc l'acte individuel se conforme aux idées de l'imaginaire social, il s'agira d'un acte stratégique; en revanche, aussitôt que l'acte individuel commence à s'approprier ces idées pour ses propres buts, à les détourner, à les modifier, à mal les comprendre, il deviendra « tactique ».

Ceci me semble être le point principal. Ce que de Certeau vise, c'est un modèle de liberté (contre un modèle de détermination): l'Homme (la Femme) n'est pas livré aux structures qui existent sans lui, en dehors de lui, mais, ces structures, il les forme, il les crée luimême, chaque fois qu'il agit, par ses arts de faire, ses manières de faire, c'est-à-dire par ses actions, son comportement qui, sciemment, inconsciemment, peuvent être conformes ou bien entièrement divergents de ce que préconise la «stratégie». Une «société » se présente donc comme la somme des actes pragmatiques individuels, et une société particulière se caractérise comme "homogène » ou bien « déchirée » par la relation entre actes stratégiques et actes tactiques, ce qui, d'ailleurs, vise la relation prémoderne entre "autorité » (stratégique) et " pluralisation » (tactique).

Cette relation est illustrée par Certeau à l'aide des deux termes « lieu » et « temps ». La stratégie appartient au paradigme « lieu », la tactique, par contre, à celui du «temps»; l'une apparaît comme «l'établissement d'un lieu», l'autre comme une «utilisation du temps » :

Les stratégies sont donc des actions qui, grâce au postulat d'un lieu de pouvoir (la propriété d'un propre), élaborent des lieux théoriques (systèmes et discours totalisants) capables d'articuler un ensemble de lieux physiques où les forces sont réparties. [...] Les tactiques sont des procédures qui valent par la pertinence qu'elles donnent au temps - aux circonstances que l'instant précis d'une intervention transforme en situation favorable, à la rapidité de mouvements qui changent l'organisation de l'espace, aux relations entre moments successifs d'un "coup », aux croisements possibles de durées et de rythmes hétérogènes, etc. 9 .

${ }^{9}$ Certeau, op. cit., p. 62sq. La « stratégie " semble donc plutôt alliée aux élites, tandis que la «tactique » semble avoir tendance à se rattacher au "peuple »; pour cette différence, voir, pour l'Angleterre, Peter Burke, Popular Culture in Early Modern Europe, Aldershot, Wildwood House, 1988, et pour la France, Robert Muchembled, Culture populaire et 
Systématique et localisation atemporelle d'une part, pragmatique et temporalité alocale de l'autre - rien ne démontre cela mieux que le champ auquel Certeau a emprunté cette idée : à Clausewitz et à son utilisation des termes « stratégie » et « tactique » dans l'art de mener la guerre. La «stratégie » abstraite est représentée par la table illustrant le modèle de la bataille, la carte imaginaire représentant le lieu, utilisant des flèches pour marquer les avances et les retraites. Les actes stratégiques ou tactiques, par contre, c'est tout ce qui a lieu, au cours de la bataille concrète, à un certain lieu, à un certain temps ; et ils apparaissent soit comme réalisation stratégique, soit comme "ruse » (metis) spontanée temporaire, soit comme «braconnage » situationnel ${ }^{10}$

Puisque une société ne peut pas être considérée comme un système, mais plutôt comme une chaîne ou une trace d'actes d'appropriation individuels, comme le résultat précaire, momentané, inachevable d'un processus incessant de production et de construction, somme fictive de toutes les actions pragmatiques, elle se caractérise par une négociation permanente ; et c'est justement cette négociation qui l'institue en tant que construction collective, comme un contrat présent toujours à renouveler ${ }^{11}$. Ce processus permanent devient de plus en plus remarquable dans les situations où la pragmatique individuelle commence à l'emporter sur la sémantique sociale, c'est-àdire dans les temps de crise, de changement, de restructuration. Voilà précisément ce qui caractérise le Londres de 1600 (ill. 2) ${ }^{12}$. Car il s'agit là d'une époque où les actions individuelles ne semblent plus tenues ni par une épistémè généralement acceptée comme celle des analogies, ni par une foi unique monologique telle que le catholicisme, ni par un ordre politique évident symbolisé par une hiérarchie sociale fixe, ni par un calendrier séparant une fois pour toutes les temps de la «vie

culture des élites dans la France moderne (XVe-XVIII siècle) [1978], coll. Champs, Paris, Flammarion, 1991.

${ }^{10}$ Ibid., p. 63sq. et p. 239 sq

${ }^{11}$ Pour la notion de " construction », voir Peter L. Berger / Thomas Luckmann, The Social Construction of Reality. A Treatise in the Sociology of Knowledge, coll. Anchor Books, Garden City, NY, Doubleday \& Co., 1967; pour la dispersion du terme " négociation ", qu'il semble avoir emprunté à Michel de Certeau, voir Stephen Greenblatt, Shakespearean Negotiations. The Circulation of Social Energy in Renaissance England, Oxford, Clarendon, 1992.

12 Pour une brève description illustrée, voir François Laroque, Shakespeare. Comme il vous plaira, coll. Découvertes Gallimard, Paris, Gallimard, 1991, chap. 2. 
sérieuse » des temps (et du «non-lieu $\left.{ }^{13} »\right)$ d'une licence carnavalesque ${ }^{14}$.

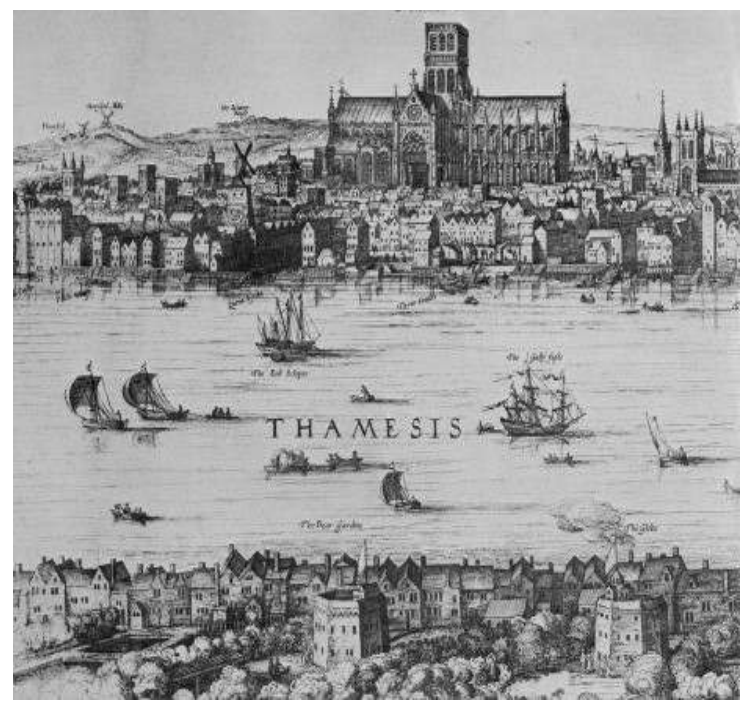

Ill. 2: Vue de Londres (J. C. Visscher, détail). Barker \& Jackson, op . cit., p. 94-95.

\section{Acteurs-réseaux et constellations}

Jusqu'ici, j’ai parlé de « lieux », de « non-lieux », et d' « espaces ». Cela implique à la fois des personnages qui s'y trouvent, qui les peuplent, c'est-à-dire des « acteurs », ou plutôt des « acteurs-réseaux ${ }^{15}$ », qui,

13 Pour le concept du «non-lieu », qu’il utilise pour l'époque de la postmodernité, voir Marc Augé, Introduction à une anthropologie de la surmodernité, coll. La librairie du Xx siècle, Paris, Seuil, 1992.

14 Pour une reconstruction du calendrier élisabéthain, et la culture renaissante du carnaval en Angleterre, voir François Laroque, Shakespeare's Festive World. Elizabethan Entertainment and the Professional Stage, trad. Janet Lloyd, Cambridge, C.U.P, 1993.

15 J'emprunte ce terme à Bruno Latour, Changer de société, refaire de la sociologie, trad. Nicolas Guilhot, Paris, La Découverte, 2007. 
avec leurs trajectoires, contribuent à leur tour à la constitution, la construction, la négociation de la société (du monde social) en question. Si une société n' « existe» pas, mais doit être considérée comme une construction imaginaire collective, si elle n'est jamais résultat, mais toujours processus, trace d'une collectivité pragmatique, une ville peut à son tour être conçue comme une telle société condensé $\mathrm{e}^{16}$. Elle se caractérise par une certaine complexité; elle apparaît comme un des foyers de la société en question; elle est marquée par les processus de dynamisation, d'accélération, de différenciation, par une certaine perte de contrôle, par la confusion, l'immensité, l'anonymat, mais aussi par la tolérance et un certain degré de respect mutuel17. Par conséquent, on peut regarder ses habitants comme les membres d'un (large) groupe et les décrire en termes de « réseau ».

Sociologiquement, un réseau se définit d'un point de vue structural par son ampleur, sa densité, c'est-à-dire par le nombre de ses membres qui se trouvent en contact direct ou indirect les uns avec les autres et par le degré général de leurs affiliations ; il se caractérise d'un point de vue sémantique par la complexité, l'intensité, et la qualité des relations entre ses membres, c'est-à-dire par une certaine univocité ou pluralité des relations, la fréquence autant que la durée des contacts, et leur résultante qualitative ${ }^{18}$. Par conséquent, un réseau étroit est marqué par un grand nombre de contacts de premier ordre, une haute densité, la pluralité des relations, une haute fréquence et une longue durée des contacts, et un bon nombre (non-négligeable) de résultats communs; il se trouve individualisé par exemple par la multiplication des relations qui fondent une habitation commune, un intérêt professionnel commun ou des relations amicales et/ou familiales, etc. ${ }^{19}$.

\footnotetext{
16 Pour l'analyse matérielle de la ville, sa physiognomie autant que son anatomie, voir les deux ouvrages magistraux de Spiro Kostof, The City Shaped, Londres, Thames \& Hudson, 1991, et The City Assembled, Londres, Thames \& Hudson, 1992.

${ }^{17}$ Voir la caractéristique de la ville chez Braudel, op cit., et les thèses de Louis Wirth, " Urbanism as a Way of Life ", in : Paul K. Hatt et A. J. Reiss, éd., Cities and Society. The Revised Reader in Urban Sociology, New York, Free Press, 1957, p. 46-63.

18 Voir Jeremy Boissevain, "Social network", in Sociolinguistics. An International Handbook of the Science of Language and Society, éd. Ulrich Ammon et al., vol. 3.1, Berlin/New York, de Gruyter, 1987, p. 164-169.

${ }^{19}$ Voir sur ce point avant tout le deuxième tome des Arts de faire : Michel de Certeau, Luce Giard et Pierre Mayol, L'invention du quotidien. 2. Habiter, cuisiner, éd. Luce Giard, coll. Folio Essais, Paris, Gallimard 1994; pour une utilisation de la notion de « réseau » dans
} 
Quant aux acteurs dans un réseau, comme l'a souligné Bruno Latour, ils ne sont pas tellement des sujets autonomes, des individus actifs qui agissent selon leur volonté, mais plutôt des instances de répartition, des «nœuds » d'interaction à qui « on », c'est-à-dire un autre acteur-réseau, « fait faire » quelque chose :

Nous voilà enfin libérés de tout un ensemble de discussions portant sur le «poids relatif» de la «liberté individuelle» par rapport à la " détermination structurelle » : chaque médiateur ponctuant une chaîne d'action est un événement individualisé parce qu'il est connecté à de nombreux autres événements individualisés. Voilà qui nous offre une bonne occasion de faire nos adieux à la notion d' «acteur » que j'ai utilisée jusqu'ici comme un figurant provisoire. Si ce terme laisse à désirer, ce n'est pas parce qu'il se réfère aux humains - nous avons appris à ignorer cette limite - mais parce qu'il désigne une source d'initiative ou un point de départ, en tout cas l'origine d'un vecteur orienté vers une fin. Bien entendu, lorsque la sociologie du social était dominante, il était important d'insister sur les acteurs, l'activité, l'initiative [...]. Mais tel n'est plus le cas avec la sociologie de l'acteurréseau : la théorie de l'action elle-même est différente, puisque nous nous intéressons maintenant à des médiateurs faisant faire des choses à d'autres médiateurs. "Faire faire " n'est pas la même chose que «causer » ou « faire » : l'expression recèle en son sein une duplication, une dislocation, une traduction qui modifient d'un coup tout l'argument. Il était auparavant impossible de relier un acteur à ce qui le faisait agir, sans être accusé de le «dominer», le «limiter» ou l'«asservir». Ce n'est plus le cas. Plus il a d'attachements, plus il existe; plus il y a de médiateurs, mieux c'est ${ }^{20}$.

Voilà la base de ce qu'on pourrait appeler une « constellation », si on comprend par là une relation particulière entre un groupe étroit de médiateurs constituant (ou bien aussi détruisant) un réseau en faisant faire à d'autres médiateurs du groupe des actes inattendus, imprévus, en leur faisant produire des "événements ${ }^{21}$ », tels qu'on peut les observer dans le Londres de Shakespeare.

l'analyse (socio)linguistique voir p. ex. l'ouvrage de Lesley Milroy, Language and Social Networks, Oxford, Blackwell, 1987.

${ }^{20}$ Latour, op. cit., p. 315sq. (c'est Latour qui souligne) ; pour la notion de «médiateur » au lieu d' " intermédiaire », et celle d' « acteur », voir, respectivement, p. 55sq. et p. 67sq.

${ }^{21}$ Pour la notion de "constellation ", utilisée pour la première fois par le philosophe allemand Dieter Henrich dans sa tentative d'expliquer l'émergence de l'Idéalisme allemand provenant d'Iéna et de Tübingen, voir les contributions - notamment celles des deux éditeurs - au tome Konstellationsforschung, éd. Martin Mulsow et Marcelo Stamm, coll. stw, Francfort-sur-le Main, Suhrkamp, 2005. 


\section{Topographies et trajectoires}

Je vais me concentrer maintenant sur l'espace londonien. La ville de Londres autour de 1600 peut être considérée comme une des premières «métropoles » européennes : elle est caractérisée par une expansion énorme ; vers 1600 on l'estime à environ 200000 habitants dans un espace relativement étroit; en même temps, on voit déjà l'émergence de ce qu'on pourrait nommer une «banlieue ${ }^{22}$ ». Par conséquent, on peut parler d'un premier grand et immense « espace urbain », d'une « urban agglomeration ${ }^{23}$ ».

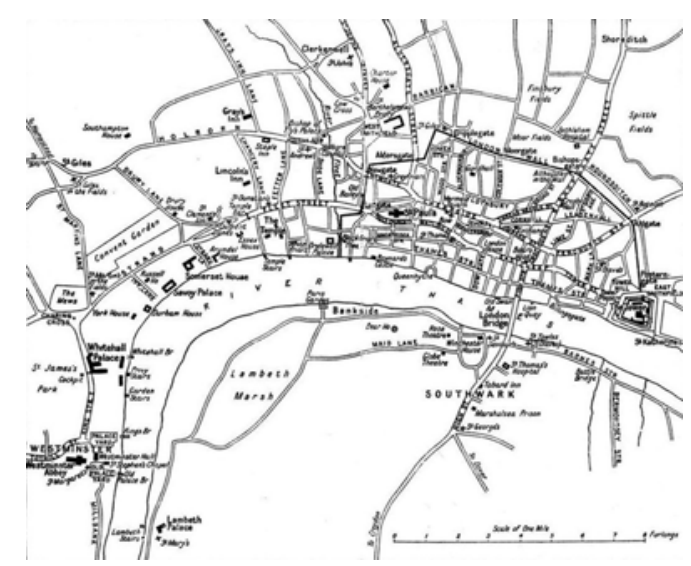

Ill. 3: Carte de Londres. - John Stow, A Survey of London, éd. H. B. Wheatley, Everyman History, Londres, Dent, 1987, p. xix.

En jetant un regard sur une carte, on découvre son ancienne topographie (ill. 3). Le Londres de Shakespeare se situe juste après le coude de la Tamise ; au Nord se trouve la City of London entourée par

22 Pour la caractérisation du Londres élisabéthain comme «métropole », comme pour l'utilisation du terme "banlieue», voir Elizabethan-Jacobean Drama. A New Mermaid Background Book, éd. G. Blakemore Evans, Londres, A \& C Black, 1987, p. 204sq. ; voir aussi les descriptions récentes (populaires) chez Bill Bryson, Shakespeare. The World as a Stage, Londres, Harper, 2008, p. 65sq., Richard Tames, Shakespeare's London on Five Groats a Day, Londres, Thames \& Hudson, 2009, ou bien littéraires comme, par exemple, chez Peter Ackroyd, London. The Biography, Londres, Chatto \& Windus, 2000, p. 89sq.

23 Peter Burke, "Popular Culture in Seventeenth-Century London », in Barry Reay, éd., Popular Culture in Seventeenth-Century England, Londres/Sydney, Croom Helm, 1985, p. 31-58, p. 33 . 
un mur (ligne noire) ; celle-ci est marquée par un centre religieux (St. Paul's) aussi bien que par un centre commercial (Cheapside/Gresham College); elle est en contact direct avec la rive droite par le pont (unique) de London Bridge. Son organisation interne (ill. 4) la divise en 24 districts (wards), dont 13 se trouvent à l'est de la petite rivière de Walbrook: Portsoken without the walls, Tower street, Aldgate, Lime street, Bishopsgate within \& without, Broad street, Cornhill, Langbourne, Billingsgate, Bridge within, Candlewick street, Walbrook, Downgate, tandis que 11 se situent à l'ouest du Walbrook: Vintry, Corwainer street, Basinghall, Cripplegate within \& without, Aldersgate within \& without, Farringdon within, Bread street, Queenhithe, Castle Baynard, un $25^{\mathrm{e}}$ se trouvant en dehors du mur: Farringdon without, plus, au sud, dans la «banlieue» de Southwark, Bridge (ward) without ${ }^{24}$.

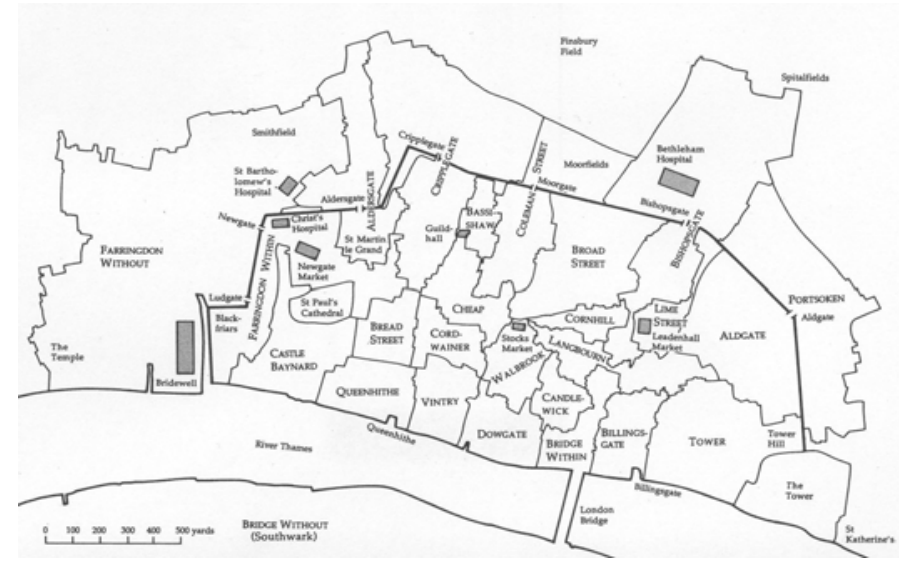

Ill. 4: Districts de Londres. - Steve Rappaport, Worlds $w$ ithin Worlds, Cambridge, C. U. P, 1989, p. 33 .

À côté de la ville de Londres, à l'ouest, se trouve une deuxième ville : celle de la City of Westminster (ill. 5), qui est à l'époque une cité à part, disposant de la cathédrale du couronnement, et qui constitue, avec la Tour à l'est, le lieu principal pour les activités de la cour (et, partant, de la noblesse) et, en plus, celles du parlement. On aurait alors déjà deux « lieux » officiels dans le Londres prémoderne : la cour et la

24 Pour la liste et une description contemporaine, voir John Stow, A Survey of London, éd. H. B. Wheatley, Everyman History, Londres, Dent, 1987, p. 109sq. 
ville. Et il faut en ajouter un troisième, qui se trouve entre la cour et la ville, dans les soi-disant «liberties », les (non-)lieux de licence appartenant ni à la juridiction municipale ni à celle de la cour, et qui transgressent les espaces respectifs de Londres et de Westminster : je parle des écoles de droit situées dans les Inns of Court ${ }^{25}$.

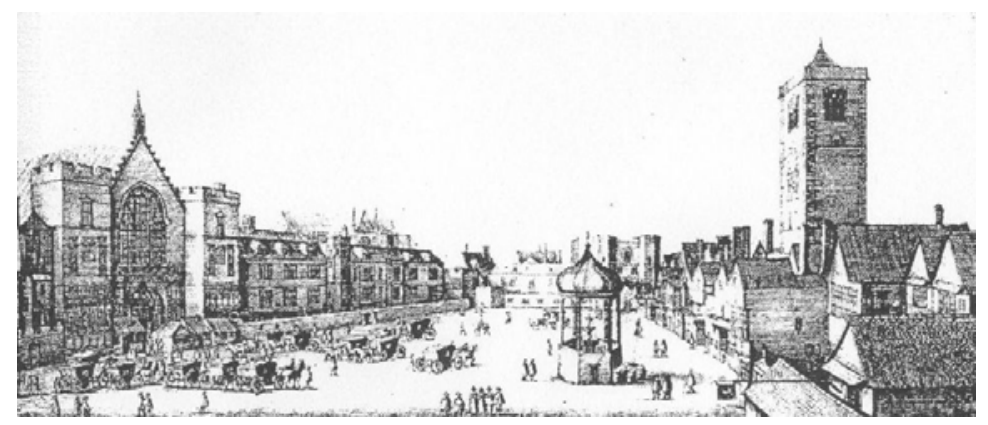

Ill. 5: Westminster (W. Hollar, détail). - The Eliza b et ha n Underw orld, éd. A. V. Judges, Londres, Routledge, 1930, p. 287.

On peut alors distinguer (au moins) trois milieux, c'est-à-dire trois espaces sociaux différents ; et on pourrait à partir de là tracer des lignes de mouvement de citoyens, d' « acteurs-réseaux » londoniens se frayant un chemin à travers ces espaces, ce qui donnerait des trajectoires, des itinéraires individuels. Par conséquent, dans la City of London même, à l'est de la Fleet, il y aurait une première ligne menant de la porte d'Aldgate à celle de Newgate : ce serait l'axe est-ouest, passant par Cornhill ou Fenchurch/Lombard Street, West Cheape, et Newgate Street avec, avant tout, St. Paul's, tandis qu'une autre ligne relierait Bishopsgate et Bridge gate, constituant l'axe nord-sud et comprenant Bishopsgate Street avec le pont de London Bridge, sans oublier, bien sûr, l'alternative de traverser la Tamise en bateau. D'autres trajectoires se dessineraient entre les deux cités par Newgate et le Strand autant que dans l'entre-deux des Inns of Court. Tout ceci signale déjà une ville extrêmement mobile, qui se voit accélérée par de nouveaux moyens de transport comme, par exemple, les coches

25 Voir Blakemore Evans, op. cit., p. 152sq. ; pour les particularités des Inns of Court au temps de Shakespeare, voir surtout Philip J. Finkelpearl, John Marston of the Middle Temple. An Elizabethan Dramatist in His Social Setting, Cambridge, MA, Harvard U.P., 1969, et Wilfrid R. Prest, The Inns of Court under Elizabeth I and the Early Stuarts, 15901640, Londres, Longman, 1972. 


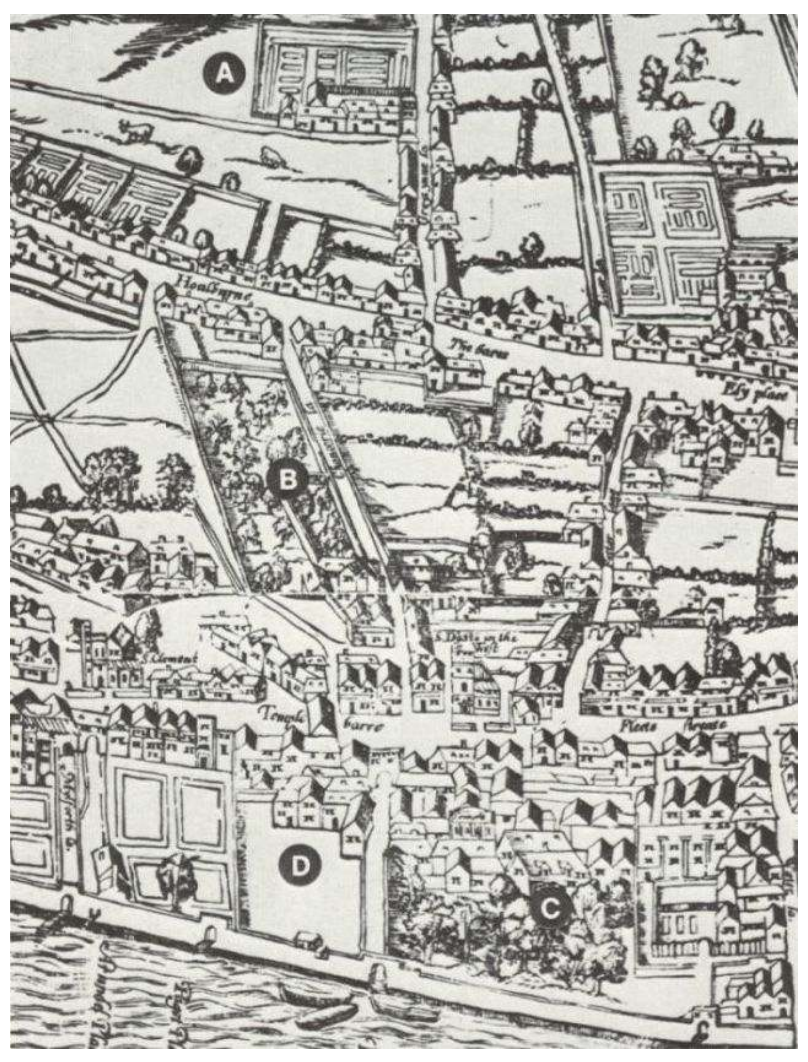

Ill. 6: Localisation des Inns (Agas, détail)

Barker \& Jackson, op. cit., p. 82. 


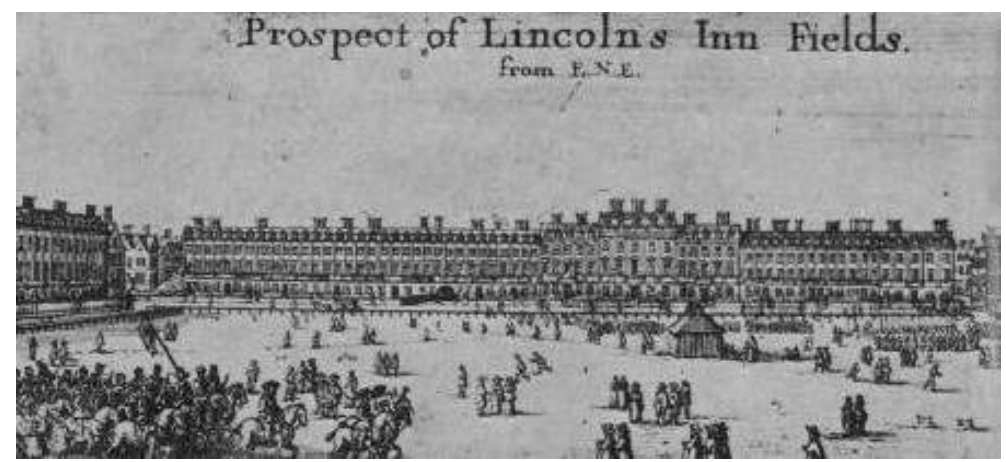

IIl. 7: Lincoln's Inn Fields (W. Hollar, détail)

Barker \& Jackson, op. cit., p. 118-119.

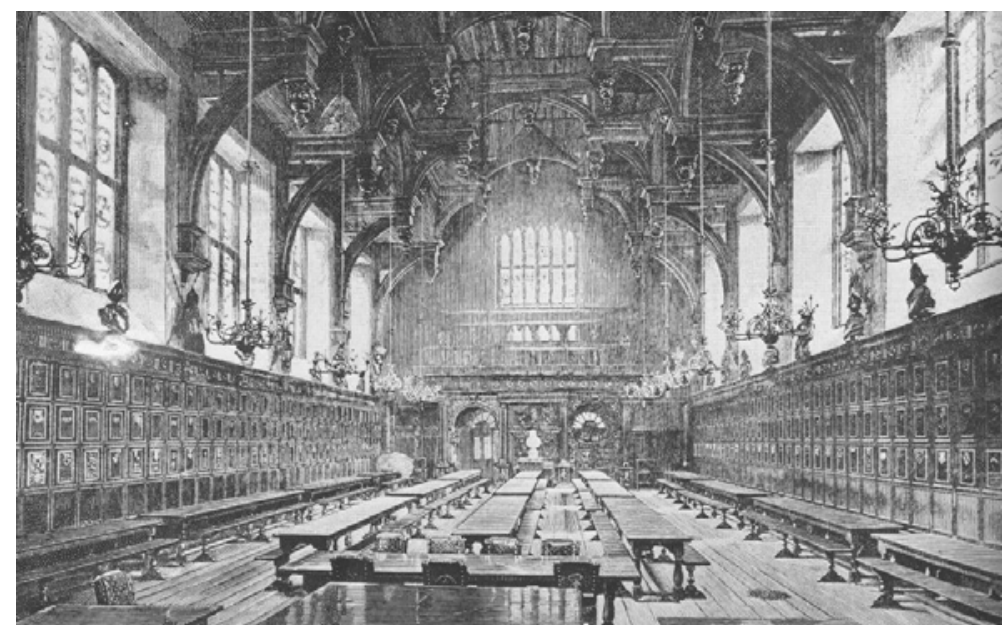

Ill. 8 : Middle Temple Hall - Barker \& Jackson, op. cit., p. 84. 
importés de l'Allemagne dans les années $1560^{26}$. Ce qu'on observe alors, ce n'est pas seulement un influx permanent venant du dehors, mais aussi un mouvement incessant à l'intérieur, ce qui me ramène aux acteurs.

\section{Urbanité et théâtralité}

Quant aux groupes sociaux, on peut aussi, en principe, en différencier trois : celui de la noblesse basé à Westminster ; celui des citoyens qui composent la cité de Londres; et le groupe des «intellectuels » caractéristique des Inns of Court ${ }^{27}$. En tant que "transformateur », ou bien comme "model of modernisation ${ }^{28}$ ", l'espace urbain semble transformer ses habitants, eux aussi, en «acteurs » se comportant comme s'ils se retrouvaient sur une (large) scène ; ce qui s'établit, est une «culture de façades ${ }^{29}$ ", une ambiance urbaine marquée par un élément de théâtralité inéluctable, dans laquelle la plupart de ses acteurs semblent convaincus que "Totus mundus agit histrionem », ou bien que "All the world's a stage, / And all the men and women merely players ${ }^{30}$. " Le Londres de 1600 ressemble à une "società spettacolo ${ }^{31} »$; l'espace londonien est un théâtre, et se trouve également représenté dans le théâtre - «Our scene is London ${ }^{2}$ »; et

\footnotetext{
${ }^{26}$ Voir Stow, op. cit., p. 77.

27 Pour cette tripartition voir, p. ex., Leonardo Benevolo, La ville dans l'histoire européenne, trad. Sophie Gherardi, Paris, Seuil, 1993, chap. 2.

${ }^{28}$ Peter Burke, Culture and Society in Renaissance Italy 1420-154O, Londres, B. T. Batsford, 1972, p. 265.

29 Pour ce terme, voir Peter Burke, The Historical Anthropology of Early Modern Italy. Essays on Perception and Communication, Cambridge, C.U.P., 1987, p. 12 ; voir aussi Andreas Mahler, «Point of Reference or Semantic Space? Functions of Venice in Early Modern English Drama », in: German Shakespeare Studies at the Turn of the Twenty-first Century, éd. Christa Jansohn, Newark, NJ, Univ. of Delaware Press, 2006, p. 161-179.

$3^{0}$ William Shakespeare, As You Like It, éd. Agnes Latham, coll. Arden, Londres, Methuen, 1975, II.vii.139-140, la citation en latin étant, bien sûr, l'inscription au-dessus de l'entrée au Globe Theatre. Pour l'idée d'une théâtralité sociale, voir Erving Goffman, The Presentation of Self in Everyday Life, Harmondsworth, Penguin, 1976; voir aussi la structuration significative de son livre en deux parties nommées « The Theatre and the World » et " The World and the Theatre » chez Blakemore Evans, op. cit.

${ }^{31}$ Voir Burke, Historical Anthropology, op. cit., p. 10 ; Burke a emprunté ce terme à Virgilio Titone, La società italiana sotto gli spagnuoli e le origini della questione meridionale, Palermo, Flaccovio, 1978, p. 116.

$3^{2}$ Ben Jonson, The Alchemist, éd. Douglas Brown, coll. New Mermaids, Londres, Ernest Benn, 1976, Prol., v. 5 ; à partir de là, on pourrait tracer toute une topographie londonienne en se référant à des localités mentionnées même seulement dans les titres, menant de Bartholmew Fair à A Chaste Maid in Cheapside.
} 
la vie londonienne semble marquée par des spectacles qui mettent en scène ou bien la cour, ou bien la City of London, ou bien les représentants des Inns of Court ${ }^{33}$. Ces écoles de droit se caractérisent comme un milieu particulier ${ }^{34}$. Il en existe quatre (ill. 6) : Gray's Inn (A), Lincoln's Inn (B), Inner Temple (C) et Middle Temple (D). On y mène une vie à part, on habite des lieux particuliers, on poursuit des activités multiples : une "activité péripatétique » dehors comme, par exemple, à Lincoln's Inn Fields (ill. 7), d'autres activités dedans comme un certain art d'habiter, de manger, de communiquer pratiqués à Middle Temple Hall (ill. 8) ${ }^{35}$.

Les écoles se situent donc entre les deux champs plus ou moins officiels de ce qu'on pourrait nommer, avec Certeau, l'invention $d u$ quotidien élisabéthain, c'est-à-dire entre la cour et la ville, «between Westminster and the city of London [...] : [...] not in the heart of the city itself, where the great confluence and multitude of the inhabitants might disturb them in their studies; but in a private place, separate and distinct by itself, in the suburbs ». Leur nom renvoie ou bien à la cour, "because the Students in them, did there, not only study the Laws, but use such other exercises as might make them the more serviceable to the King's Court ", ou bien à la justice, à cause de leur localisation « near to the Courts of Justice aforesaid, that the students, at their leisure, may daily and duly attend, with the greatest ease and convenience $3^{36}$ ». Cependant, vers 1600 , comme on peut le voir sur la carte, les Inns of Court ne se trouvent plus tellement à part, dans la banlieue, mais plutôt déjà dans la ville : in the middle, entre la City dominée par un magistrat puritain et Westminster caractérisé par la

\footnotetext{
33 Pour la théâtralité de la cour, voir, par exemple, Blakemore Evans, op. cit., p. 175sq. ; pour la théâtralité de la City, comme par exemple, le «Lord Mayor's show, an annual spectacular procession accompanying the installation of a new mayor on October 29 ", voir Gail Kern Paster, The Idea of the City in the Age of Shakespeare, Athens, GA, Univ. of Georgia Press, 1985, p. 124sq., la citation p. 124. Pour Shakespeare et Londres, voir aussi Leah S. Marcus, Puzzling Shakespeare. Local Reading and its Discontents, Berkeley/Los Angeles, CA, Univ. of California Press, 1988.

34 Pour le suivant, voir Finkelpearl, op. cit., surtout p. 1-80.

35 Pour la classification d'activités comme « habiter », « circuler », « parler », « lire », "faire le marché ou la cuisine » comme "ruses », comme des "surprises tactiques : bon tour du "faible" dans l'ordre établi par le "fort" », voir Certeau, op. cit., vol. 1, p. 65.

${ }^{36}$ Sir John Fortescue, De Laudibus Legum Angliae, trad. Andrew Amos, Cambridge, J. Smith, 1825, p. 178sq. (c'est Fortescue qui souligne) ; voir aussi Finkelpearl, op. cit., p. 4.
} 
culture de la noblesse, dans l'entre-deux entre une culture bourgeoise et une culture cérémoniale, précisément à l'in-between ${ }^{37}$.

Aux Inns appartiennent aussi les Inns of Chancery, à l'origine les habitations des employés, mais vers 1600 plutôt les «classes préparatoires " pour cette "troisième université » que constituent les Inns of Court. C'est ainsi que Thavie's (Davy's) et Furnivall's Inn appartiennent à Lincoln's Inn, Clifford's, Clement's et Lyon's Inn à l'Inner Temple, New Inn et Strand Inn au Middle Temple et Staple Inn et Barnard's Inn à Gray's Inn. À première vue, les Inns apparaissent donc comme un «lieu » londonien dans le sens de Certeau : une place fixe d'intérêts stratégiques dans l'ensemble des pratiques sociales renaissantes. Elles sont organisées à la manière de corporations ou de guildes ${ }^{38}$. Vers 1600 , le nombre de leurs membres s'élève à environ 1000 , avec les Inns of Chancery à 1700 , ce qui n'est pas vraiment beaucoup par rapport aux 200000 habitants estimés ; cependant, en ce qui concerne leur influence, leur visibilité en ville, elles représentent, aussi par rapport à la cour, le «largest single group of literate and cultured men in London ${ }^{39}$ », c'est-à-dire, une force intellectuelle qui ne doit pas être sous-estimée. Ce groupe se recrute souvent dans la noblesse de "souche " (landed gentry), mais aussi dans les familles engagées dans le commerce; ses membres sont en général dotés d'une éducation secondaire (grammar school), et même supérieure, ayant déjà fait leurs études à Oxford ou à Cambridge avant de passer à Londres; et ils sont assez « riches » pour pouvoir payer les 28 livres de droits d'admission. C'est exactement ce mélange qui semble constituer un milieu innovateur caractéristique pour le développement de nouvelles idées, pour une certaine "explosion» de créativité déclenchée par un nombre d'« acteurs-réseaux », de « go-betweens ${ }^{40}$ »,

\footnotetext{
37 Pour la situation autour de 1600 , voir Stow, op. cit., p. 70sq. ; pour une caractérisation de l'époque élisabéthaine comme " a period "between two worlds" ", et les théâtres de Southwark comme " inside / outside space », voir Margot Heinemann, " Political drama », in The Cambridge Companion to English Renaissance Drama, éd. A. R. Braunmuller et Michael Hattaway, Cambridge, C.U.P., 1990, p.161-205, p. 162, et Richard Wilson, Will Power, Essays on Shakespearean Authority, Londres, Harvester Wheatsheaf, 1993, p. 41. 38 À ce sujet, voir aussi la contribution de Christophe Hausermann.

39 Finkelpearl, op. cit., p. 5 .

${ }^{40}$ Voir, à ce sujet, les contributions dans Renaissance Go-Betweens. Cultural Exchange in Early Modern Europe, éd. Andreas Höfele et Werner von Koppenfels, Berlin/New York, de Gruyter, 2005 .
} 
de «médiateurs » inattendus «faisant faire » à d'autres acteurs des choses imprévues ${ }^{41}$.

L'éducation juridique dure au moins sept ans. Elle est divisée en trois phases et se trouve hiérarchisée selon ce qu'on pourrait appeler, avec Certeau, un «art de manger » : en tant que Inner Barrister, on mange tout d'abord aux tables réservées pour les freshmen aux Clerk's Commons; au bout de deux ans, on accède aux tables des Master's Commons; et on finit ses études après sept ans comme Utter (Outer) Barrister pour enfin travailler soit comme tel en dehors des Inns soit comme Bencher ou Reader dans les Inns même. Le pourcentage d'étudiants réussissant à l'examen s'élevait à environ $15 \%$; c'est-à-dire, le vrai intérêt résidait moins dans la juridiction que dans l'entourage, moins dans le caractère universitaire que dans celui d'une « finishing school », moins dans la stratégie que dans les tactiques : «The ideal persisted of producing not merely a well-trained lawyer but a True Gentleman ${ }^{42}$.»

La vie aux Inns se trouve divisée en trois périodes : la période des assises, Sessions ou Terms ; la période des enseignements pendant les Grand Vacations (des tribunaux) ; et les temps de fête et de licence, les Revels, chaque période étant portée par un certain groupe, «the benchers [...] govern[ing] in term time, the barristers in vacation, and the gentlemen in the Christmas ${ }^{43}$ ». Il y a donc une phase de professionnalisme sérieux, une phase d'appropriation, de, pour ainsi dire, «théâtralité » sérieuse des normes juridiques, et une phase de mascarade joyeuse, les deux premières phases représentant la "stratégie » dans le sens d'une acceptation ou appropriation du discours juridique, comme affirmation d'une discipline; la dernière fonctionnant, par contre, comme une phase de soulèvement, de suspension non-discursive, dans le sens de «tactiques » potentiellement « anti-disciplinaires 44 ».

C'est ainsi que le «lieu » des Inns se transforme en un «lieu pratiqué », et non seulement pratiqué, mais pratiqué différemment : il

${ }^{41}$ Pour une explication de la base sociologique de la créativité renaissante, faisant surtout référence au groupe des "ingénieurs-artisans » proposé par Edgar Zilsel, voir Wolfgang Krohn, «Zur soziologischen Interpretation der neuzeitlichen Wissenschaft », in Edgar Zilsel, Die Ursprünge der neuzeitlichen Wissenschaft, éd. Wolfgang Krohn, coll. stw, Francfort-sur-le-Main, 1976, p. 7-43, p. 23sq.

42 Finkelpearl, op. cit., p. 10sq.

43 State Papers Domestic, Charles I, 1639-1640, p. 304 ; cité d'après ibid., p. 36.

44 Certeau, op. cit., vol. 1, p. XL. 
se transforme en un « $\operatorname{espace}^{45}$ ». Ce qui s'ouvre, ce sont des possibilités d'inventions (dans le sens certalien) individuelles pluralistes - du quotidien, des utilisations pragmatiques individuelles du système social. Du point de vue stratégique, ceci se trouve entamé tout d'abord à travers l'instruction même, c'est-à-dire à l'aide de discours et de débats. Le devoir d'un Reader, par exemple, était, bien sûr, « to read, expound, and declare some Estatute openly unto all the Company of the House ${ }^{46}$ », donc un exercice avant tout monologique, dans lequel un texte juridique était lu, expliqué, et puis discuté par ordre de séniorité, de l’Utter Barrister le plus jeune jusqu'au Bencher le plus vieux selon un système de questions et de réponses, avant que le Reader n'énonce le dernier mot autoritaire comme conclusion. D'autre part, il y avait les «moots», exercices dialogiques qu'on pourrait appeler des mock disputations, et qui avaient lieu chaque soir dans les Grand Vacations, et pendant la période des Terms deux fois par semaine. Ils consistaient en une explication publique d'un cas « in homely Law-French 47 » par un étudiant, accompagnée par les pours et les contres prononcés par deux Utter Barristers et commentée enfin en anglais par les trois Benchers menant le tribunal. Une autre variante de ceci est le "bolting » associé surtout avec Gray's Inn, où, chaque jour après le dîner et après le souper, trois Inner Barristers et trois Utter Barristers entraient dans un jeu juridique les uns contre les autres. En plus, pendant la période des Terms, il fallait assister aux assises officielles de Westminster et de Guildhall à des places réservées exclusivement aux étudiants des Inns (nommées «le cribbe $\left.{ }^{48} »\right)$; et ceci se trouvait complété par la lecture régulière et systématique des collections de «cas» bien connues sous les noms de leurs auteurs Brooke, Perkins, Plowden, Littleton et Dyer.

On voit bien que les Inns doivent être conçues comme un lieu de dialogicité, sinon de plurivocité, mélangeant les textes juridiques en latin, la langue juridique anglo-normande et l'anglais comme langue

\footnotetext{
45 Ibid., vol. 1, p. 173.

46 Finkelpearl, op. cit., p. 9 .

47 William Dugdale, Origines Juridiciales, or, Historical Memorials of the English Laws, Courts of Justice, Forms of Tryal, Punishment in Cases Criminal [...], Londres, Thomas Newcomb, 1671, p. 194 ; cité d'après ibid., p. 9.

48 Ibid.
} 
maternelle quotidienne ${ }^{49}$. En plus, ils constituaient un lieu du débat, de la parole libre ; c'est-à-dire, ce qui se mélangeait, ce n'étaient pas seulement les langues, mais aussi les points de vue idéologiques, comme le démontre très bien l'activité simultanée du calviniste Walter Travers et de l'anglican Richard Hooker dans leurs rôles de Masters of the Temple en 1585: "Here the pulpit spake pure Canterbury in the morning, and Geneva in the afternoon $5^{0}$ ». Leurs membres étaient connus partout à la ronde, comme l'illustre le portrait suivant :
Publius student at the common law,
Oft leaves his bookes, and for his recreation,
To parish garden doth himselfe withdraw,
Where he is ravisht with such delectation,
As downe amongst the dogges and beares he goes,
Where whiles he skipping cries to head to head,
His sattin doublet and his velvet hose,
Are all with spittle from above bespread.
Then is he like his fathers country Hall,
Stinking with dogges, and muted all with hawkes,
And rightly too, on him this filth doth fall,
Which for such filthy sports his bookes forsake,
Leaving old Ployden, Diar, and Brooke alone,
To see old Harry Hunkes and Sakersone ${ }^{51}$.

Et, dans la même veine, le portrait de l'étudiant des Inns dans The Young Gallants Whirligigg de Francis Lenton :

No, no, good man, hee reades not Littleton,

But Don Quix Zot, or els The Knight of the Sun :

And if you chance unto him put a Case,

Hee'll say perhaps you offer him disgrace. [...]

Instead of Perkins pedlers French, he sayes

He better loves Ben : Johnsons booke of Playes,

\footnotetext{
49 Pour la notion d’une «dialogicité », ou "plurivocité », prémoderne, voir Mikhaïl Bakhtine, L'Euvre de François Rabelais et la culture populaire au Moyen Âge et sous la Renaissance, trad. Andrée Robel, coll. tel, Paris, Gallimard, 1985.

$5^{0}$ Thomas Fuller, History of the Worthies of England, éd. P. Austin Nuttall, Londres, New Edition, 1840, vol. 1, p. 423 ; cité d'après Finkelpearl, op. cit., p. 62sq

${ }^{51}$ The Poems of Sir John Davies, éd. Clare Howard, New York, Columbia U. P., 1941, p. 55sq.; pour les ours appelés Sackerson et Harry Hunkes, voir Andreas Höfele, "Sackerson the Bear», REAL, $\mathrm{n}^{\circ}$ 17, 2001, p. 161-177. Voir aussi Sir Thomas Overbury, "A Fantastic Inns of Court Man ", in London in the Age of Shakespeare. An Anthology, éd. Lawrence Manley, Londres \& Sydney, Croom Helm, 1986, p. 320 sq.
} 
But that therein of wit he findes such plenty,

That hee scarce understands a jest of twenty ${ }^{2}$.

Comme l'indiquent les références au Don Quichotte de Cervantès ou aux drames de Ben Jonson, la grande majorité des étudiants s'intéressait à bien d'autres choses qu'à la simple appropriation stratégique de connaissances juridiques. Il s'agissait là, en premier lieu, d'un intérêt dirigé vers les appâts de la ville et aussi, en raison de la plurivocité mentionnée ci-dessus, vers la littérature. Dans les années 1558-1572, mais aussi plus tôt et plus tard, les Inns figuraient comme l'un des plus grands centres de traduction du pays, associés avec des noms tels que Jasper Heywood, George Turberville, Barnabe Googe, Gascoigne, Sackville and Norton, North, Lodge, Fraunce, Raleigh, Harington, Campion, Donne, Bacon, Davies, Marston, Ford ou Beaumont 53 .

Mais l'intérêt littéraire se trouve surtout actualisé, comme mentionné, dans les Revels, et avant tout « in the Christmas 54 ». Par «Revels" on comprenait des fêtes carnavalesques ${ }^{55}$, dans la nomenclature de Certeau des «utilisations du temps » ou bien des « espaces » libres, c'est-à-dire l'option d'agir, pour un moment, de façon tactique dans le cadre d'un sérieux avant tout stratégique. Ils s'opposent aux Grand Vacations, et ils sont marqués par une déhiérarchisation, par la licence temporaire d'une suspension de la sémantique officielle du groupe, tout en sachant, bien sûr, que cette suspension ne pourra pas subsister pour toujours. Les Christmas Revels commençaient par l'élection d'un « Prince », c'est-à-dire d'un roi carnavalesque ou lord of misrule juste avant Noël. À Gray's Inn, celui-ci s'appelait (selon la paroisse) « Prince of Purpoole» (Portpool), à Lincoln's Inn «Prince de la Grange », à l'Inner Temple «Prince of Sophie » et au Middle Temple «Prince d'Amour». Après son intronisation s'ensuivait le recrutement d'un entourage de courtisans, accompagné par une procession introduisant le nouveau Prince chez

52 Francis Lenton, The Young Gallants Whirligigg : or Youthes Reakes, Londres, 1629, p. 4 ; cité d'après Finkelpearl, op. cit., p. 13sq.

53 Voir ibid., p. 19; pour l'importance du terme «traduction" dans une sociologie d'« acteurs-réseaux », voir Latour, op. cit., p. 152sq.

54 Voir Laroque, Festive Comedy, op. cit., p. 74sq.

55 Pour la notion du «carnaval », voir Bakhtine, op. cit., et le petit livre de Daniel Fabre, Carnaval ou la fête à l'envers, coll. Découvertes Gallimard, Paris, Gallimard, 1992. Voir aussi W. R. Elton, Shakespeare's Troilus and Cressida and the Inns of Court Revels, Aldershot, Ashgate, 2000. 
les voisins, accordant des « knighthoods » et établissant le règne par des commandes et des lois fictives garantissant l'autorité du Prince jusqu'à la fin de son règne le mercredi des Cendres ou à la Chandeleur.

C'est surtout dans les années 1590 qu'on observe une certaine transgression des Revels. Les tactiques commencent à prendre le dessus, c'est-à-dire, elles ne restent plus dans le cadre festif réservé au carnaval, mais prétendent à une certaine valeur sérieuse. Deux fêtes particulières se trouvent très bien documentées : les Gesta Grayorum fêtés l'hiver 1594 / 95 et les Revels du Prince d'Amour au Middle Temple datant de 1597 / 9856. Elles sont avant tout concentrées sur les trois semaines entre le 24 décembre et le 14 janvier où il y a un programme de fête très détaillé et des activités festives permanentes à tous les niveaux. Une des fonctions principales réside dans la stabilisation identitaire du groupe ; les occasions, les effets, la durée et les mises-en-scène individuelles dépendent, bien sûr, de la personne du Prince élu, de son engagement, de ses idées, de son investissement financier, mais elles dépendent également du voisinage et de la connaissance mutuelle des membres du groupe, c'est-à-dire du type de « réseau » en question. De tels réseaux se laissent reconstruire; on peut constater de multiples liens entre les membres d'une seule Inn, une certaine complexité dans la structure du groupe, due à l'habitation commune, au même champ de profession, à des relations non seulement amicales, mais familiales, et même "dynastiques ", à l'homosocialité du groupe, etc. En plus, il y a, à l'extérieur, des jumelages entre, par exemple, Gray's Inn et Inner Temple, d'une part, comme entre Lincoln's Inn et Middle Temple, de l'autre57, comme, à l'intérieur, des solidarités de membres de familles, de camarades de classe, des amis avec lesquels on a fait ses études à Oxford ou à Cambridge. Tout cela aide à former des «constellations » très particulières.

On peut tracer une telle constellation pour les années 1580 , et surtout 1590, à Middle Temple : il s'agit d'un groupe qui se connaît déjà depuis la Grammar School à Winchester ${ }^{5}$. Il comprend John Owen, futur épigrammatiste en latin, John Hoskins, rhétoricien, Henry Wotton, ambassadeur, Thomas Bastard, ecclésiastique, et John Davies,

\footnotetext{
$5^{6}$ Voir Finkelpearl, op. cit., p. 32sq.

57 Voir ibid., p. 39.

$5^{8}$ Voir ibid., p. 46sq.
} 
poète déjà mentionné ci-dessus. Les quatre premiers se transfèrent au New College à Oxford, Davies au Queen's College; là, ils font la connaissance du futur poète John Donne ; ils établissent un premier contact avec Richard Martin, qui deviendra, à partir des années 90, un des personnages principaux, et «leading wit », de Middle Temple, et plus tard le chroniqueur (Recorder) de Londres, comme avec Benjamin Rudyerd, plus tard connu pour son travail documentaire sur les Inns. À l'exception de Bastard et Owen, qui continuent leurs études à Inner Temple, le groupe entier se retrouve plus tard à Middle Temple, où ils sont rejoints par Charles Best, futur poète et juriste. C'est ici le moment où le groupe entre en contact avec le cercle autour de Ben Jonson, « the Tribe or Sons of Ben », l'auteur dramatique et rival de Shakespeare, qui va, on le sait bien, dédier sa comédie satirique Every Man out of his Humour de 1599 dans son édition prestigieuse de ses Works en 1616 aux « Noblest Nurseries of Humanity and Liberty in the Kingdom, the Inns of Court 59 ». En plus, on renouvelle, par Lincoln's Inn, le contact avec ce qu'on a appelé «the School of Donne ${ }^{60}$ ». Tout ceci pourrait avoir été la base du groupe légendaire dont on présume qu'ils ont discuté des questions de poétique à l'intérieur de la Mermaid Tavern.

Dans les années 1590, on peut donc observer comment un réseau "stratégique » d'étudiants de droit à Middle Temple se transforme lentement en une "constellation » très particulière de jeunes hommes, qui se caractérise par un bon nombre de traits communs additionnels :

To a remarkable degree, these Middle Temple wits came from the same background, pursued the same course in life, shared the same tastes and preferences, and often tended to act in very similar ways. First we should note [...] that they all became lawyers, most of them highly distinguished ones. None of them had much money; consequently they had to acquire a profession. [...] They may have been revellers, but they had to be serious students as well. Second, most of them were

\footnotetext{
59 Ben Jonson, Every Man Out of his Humour, in The Complete Plays, éd. G. A. Wilkes, Oxford, O.U.P., 1981sq., vol. 3, "Dedication »; voir aussi la référence au temps carnavalesque, " when the gown and cap is off, and the Lord of Liberty reigns » (v. 16sq.) ; pour le contexte, voir Hanna Scolnicov, Experiments in Stage Satire. An Analysis of Ben Jonson's Every Man out of His Humour, Cynthia's Revels and Poetaster, Francfort-sur-leMain et al., Lang, 1987.

${ }^{60}$ Voir A. Alvarez, The School of Donne, Londres, Chatto \& Windus, 1961, surtout p. 187sq. (« Donne's Circle»).
} 
troublemakers, more or less serious disciplinary problems, and not merely as youths ${ }^{61}$.

C'est surtout ce trait anti-disciplinaire qui peut servir d'indice d'un changement de comportement. Ce qui commence à se modifier, c'est la mentalité du groupe. Le contrôle externe aussi bien que le contrôle interne du comportement social semblent changer. On s'accorde une licence qui ne reste plus dans les frontières des Revels. Le «lieu » d'une «stratégie » sérieuse entamée par un réseau se transforme en un « espace» de «tactiques » constellatives. Ceci semble être le chemin qui mène d'une simple agglomération de «lieux » à un « espace » émergeant d'une «constellation» particulière, caractérisée par des individus et leurs «trajectoires » respectives: il mène à ce qu'on pourrait appeler un « espace créateur».

Andreas MAHLER Karl-Franzens-Universität Graz

\footnotetext{
${ }^{61}$ Finkelpearl, op. cit., p. 46sq.
} 